\title{
Majorana States in Presence of Electron Interactions: Spinful Fractional Josephson Junction with a Quantum Dot
}

\author{
P. STEFAŃSKI* \\ Institute of Molecular Physics of the Polish Academy of Sciences \\ ul. Smoluchowskiego 17, 60-179 Poznań, Poland
}

\begin{abstract}
We consider a fractional Josephson junction mediated by a quantum dot in which the Zeeman field arising from the magnetic fields driving left and right wires into topological phase can be tuned. Both fields, forming an angle $\Theta$, can be rotated in the common plane perpendicular to the spin-orbit field in the wires. For $\Theta=0$ the dot can be regarded as effectively non-interacting due to the large Zeeman splitting, whereas for $\Theta \lesssim \pi$ electron interactions are switched on the dot, affecting Majorana states. The tunnel electrode, weakly coupled to the dot from the top, allows to probe their density of states via conductance measurement. We show that electron interactions renormalize Majorana peak and introduce characteristic asymmetry in the gate voltage dependence of the transverse zero-bias conductance through the dot.
\end{abstract}

DOI: 10.12693/APhysPolA.133.362

PACS/topics: 73.63.-b, 73.21.La, 31.15.V-, 74.50.+r

\section{Introduction}

Majorana fermions, exotic quasiparticles being their own anti-particles, have been recently spotted in solid state. Their properties are interesting both from fundamentals as well as their promise of fault-tolerant quantum computation [1]. In solid state systems, Majorana bound states (MBS) exhibit spin properties in the sense that, if coupled to a non-topological object, they pick a definite spin polarization of electron tunneling into them. Recent experiments of STM spin polarized tunneling into Majorana mode at the vortex center of a topological superconductor [2] show that the Majorana mode picks the spin of electron which is parallel to the external magnetic field. It is manifested in a substantially higher zero-bias peak in differential conductance as compared to the case of the antiparallel STM tip polarization and external magnetic field. Thus, equal spin Andreev reflections are favored in the transport [3].

In our work we address the question how spin dependent tunneling can be manifested in Josephson junction hosting MBS and mediated by a non-topological object (quantum dot-QD). Our device under consideration, shown in Fig. 1, is similar to that realized experimentally by Chang et al. [4]. The wires are strongly coupled through the dot electrostatically created from the part of the wire. It allows the formation of a bound fermionic state inside the dot out of the hybridized MBS adjacent to the dot.

\section{The model and theoretical approach}

We assume that the quantum dot is exposed to the action of the magnetic field $\boldsymbol{B}_{t o t}=\boldsymbol{B}_{L}+\boldsymbol{B}_{R}$ composed of magnetic fields $\boldsymbol{B}_{L}$ and $\boldsymbol{B}_{R}$ (assuming $\left|\boldsymbol{B}_{L}\right|=$

*corresponding author; e-mail: piotrs@ifmpan.poznan.pl

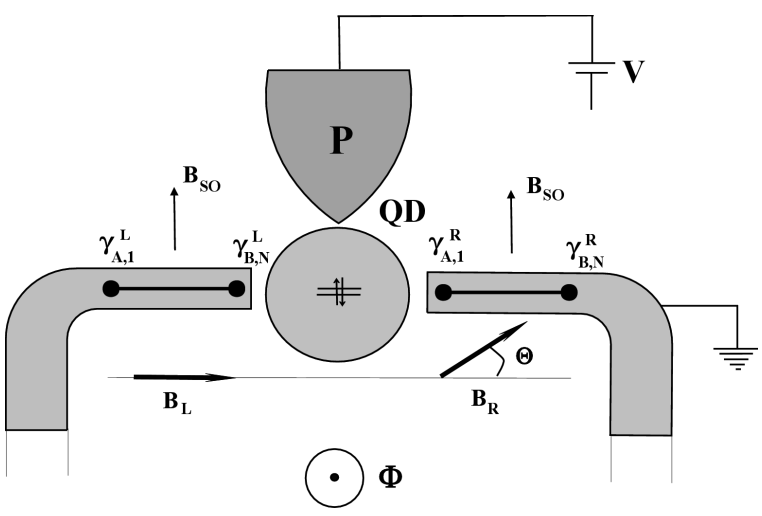

Fig. 1. Schematic of the device setup showing the rotation of magnetic fields $B_{L}$ and $B_{R}$ in the plane perpendicular to spin-orbit field $B_{S O}$, equal in both sections of the wire.

$\left|\boldsymbol{B}_{R}\right| \equiv B$ ) forming an angle $\Theta$, which drive the left and the right wire into topological state, respectively. Zeeman field inside the dot is $E_{z}=|g| \mu_{\mathrm{B}} B_{t o t}(\Theta) / 2$ with $B_{\text {tot }}=2 B \cos (\Theta / 2)$. It yields $E_{z}=E_{z}^{0} \cos (\Theta / 2)$ with $E_{z}^{0}=|g| \mu_{\mathrm{B}} B$. Under the action of Zeeman field QD level is split: $\epsilon_{\uparrow / \downarrow}=\epsilon_{d} \bar{\mp} E_{z}^{0} \cos (\Theta / 2)-V_{g}$ with the capacitative shift of the dot level by gate voltage $V_{g}$.

The dot Hamiltonian is of the form: $H_{Q D}=$ $\sum_{\sigma=\uparrow, \downarrow} \epsilon_{\sigma} d_{\sigma}^{\dagger} d_{\sigma}+U n_{\downarrow} n_{\uparrow}$. We assumed $\epsilon_{\downarrow / \uparrow}=\epsilon_{F}(\equiv 0)$ for $B=V_{g}=0$. In the following we take the induced superconducting gap $\Delta$ as energy unit, and $E_{z}^{0}=2 \Delta$ [5].

The polarization of the tunnel electrode is described by spin-dependent broadenings of the QD level: $\Gamma_{\downarrow}=$ $\Gamma_{p} \cos ^{2}(\Theta / 4)$ and $\Gamma_{\uparrow}=\Gamma_{p} \sin ^{2}(\Theta / 4)$. For $\Theta=0$, when the electrode is fully spin-down polarized, we introduced small broadening $\Gamma_{\uparrow}=10^{-6}$ for consistency of numerical calculations of the dot occupancies.

The Hamiltonian describing the tunnel electrode as well as the tunneling between the dot and the electrode 
has the usual form [6]. The topological wires are are modelled by Kitaev chains in the simplest limit of $\mu_{\alpha}=0$ and $\left|\Delta_{\alpha}\right|=t_{\alpha} \equiv t$, possessing Majorana bound states at their ends [6]; for $\alpha$-wire they are $\gamma_{A, 1}^{\alpha}$ and $\gamma_{B, N}^{\alpha}$, where $N$ is a number of sites in a given chain (see also Fig. 1).

The dot level $\epsilon_{d}$ is coherently coupled to the end-wire MBS: $\gamma_{B, N}^{L}$ and $\gamma_{A, 1}^{R}$, which are converted into the Dirac fermion $d$ inside the dot:

$$
d=\frac{1}{2}\left(\gamma_{A, 1}^{R}+\mathrm{i} \gamma_{B, N}^{L}\right) .
$$

Depending on the angle $\Theta$ formed by magnetic fields $\boldsymbol{B}_{L}$ and $\boldsymbol{B}_{R}$, the Majorana states $\gamma_{B, N}^{L}$ and $\gamma_{A, 1}^{R}$ can pick electrons with different spin orientations tunneling from the dot:

$$
d=\cos \left(\frac{\Theta}{4}\right) d_{\downarrow}+\sin \left(\frac{\Theta}{4}\right) d_{\uparrow} .
$$

From Eqs. (1) and (2) the Majorana states are calculated:

$$
\begin{aligned}
& \gamma_{B, N}^{L}= \\
& \quad \mathrm{i}\left[\cos \left(\frac{\Theta}{4}\right)\left(d_{\downarrow}^{\dagger}-d_{\downarrow}\right)+\sin \left(\frac{\Theta}{4}\right)\left(d_{\uparrow}^{\dagger}-d_{\uparrow}\right),\right] \\
& \gamma_{A, 1}^{R}=\cos \left(\frac{\Theta}{4}\right)\left(d_{\downarrow}^{\dagger}+d_{\downarrow}\right)+\sin \left(\frac{\Theta}{4}\right)\left(d_{\uparrow}^{\dagger}+d_{\uparrow}\right) .
\end{aligned}
$$

The next step is derivation of the Hamiltonian describing tunneling between topological wires via the dot. It proceeds similarly as in [6]. We retain here only the most important term describing the tunneling between MBS adjacent to the dot: $H_{\text {tun }}=-\frac{i}{2}\left(t_{L}+\right.$ $\left.t_{R}\right) \cos \left(\frac{\Delta \phi}{2}\right) \gamma_{B, N}^{L} \gamma_{A, 1}^{R}$, which with the help of Eqs. (3) and (4) it takes the refermionized form:

$$
\begin{aligned}
& H_{\text {tun }}=-\left(t_{L}+t_{R}\right) \cos \left(\frac{\Delta \phi}{2}\right) \\
& \times\left[\cos ^{2}\left(\frac{\Theta}{4}\right)\left(d_{\downarrow}^{\dagger} d_{\downarrow}-\frac{1}{2}\right)+\sin ^{2}\left(\frac{\Theta}{4}\right)\left(d_{\uparrow}^{\dagger} d_{\uparrow}-\frac{1}{2}\right)\right. \\
& \left.\quad+\sin \left(\frac{\Theta}{4}\right) \cos \left(\frac{\Theta}{4}\right)\left(d_{\uparrow}^{\dagger} d_{\downarrow}+\text { h.c. }\right)\right]
\end{aligned}
$$

In the following we will also take into account a possibility of a finite hybridization $\epsilon_{\alpha} \sim \mathrm{e}^{-L_{\alpha} / \xi}\left(L_{\alpha}\right.$ is the length of the wire and $\xi$ is the superconducting coherence length) described by $H_{h y b}=-\sum_{\alpha=L, R} \mathrm{i}\left(\epsilon_{\alpha} / 2\right) \gamma_{A, 1}^{\alpha} \gamma_{B, N}^{\alpha}$. Using Eqs. (3), (4) it has the form:

$$
\begin{gathered}
H_{h y b}=\frac{\epsilon_{L}}{2}\left[\cos \left(\frac{\Theta}{4}\right)\left(f_{L \downarrow}^{\dagger} d_{\downarrow}^{\dagger}-f_{L \downarrow}^{\dagger} d_{\downarrow}+\text { h.c. }\right)\right. \\
\left.+\sin \left(\frac{\Theta}{4}\right)\left(f_{L \uparrow}^{\dagger} d_{\uparrow}^{\dagger}-f_{L \uparrow}^{\dagger} d_{\uparrow}+\text { h.c. }\right)\right] \\
-\frac{\epsilon_{R}}{2}\left[\cos \left(\frac{\Theta}{4}\right)\left(f_{R \downarrow}^{\dagger} d_{\downarrow}^{\dagger}+f_{R \downarrow}^{\dagger} d_{\downarrow}+\text { h.c. }\right)\right. \\
\left.+\sin \left(\frac{\Theta}{4}\right)\left(f_{R \uparrow}^{\dagger} d_{\uparrow}^{\dagger}+f_{R \uparrow}^{\dagger} d_{\uparrow}+\text { h.c. }\right)\right],
\end{gathered}
$$

where $f_{\alpha \sigma}=(1 / 2)\left(\gamma_{A, 1, \sigma}^{\alpha}+\mathrm{i} \gamma_{B, N, \sigma}^{\alpha}\right)$ is the extended, non-local fermion emerging from the hybridization of two MBS. The spin index attached to Majorana operators has here the sense of Majorana spin polarization, indicating that when a Majorana state is coupled to non-topological object it can absorb or emit electrons with the specific spin projection. Moreover, the conservation of spin in Eq. (6) in tunneling processes between the dot and extended fermionic states in the wires reflects the promotion of equal spin Andreev reflections [3].

The retarded QD Green function, $G\left(t-t^{\prime}\right)=-\mathrm{i} \theta(t-$ $\left.t^{\prime}\right)\left\langle d(t) d^{\dagger}\left(t^{\prime}\right)+d^{\dagger}\left(t^{\prime}\right) d(t)\right\rangle$ is obtained by equation of motion method, utilizing refermionized Hamlitonian of the system. It has the form in $\omega$-domain for spin-down component:

$$
G_{\downarrow}(\omega)=
$$

$$
\begin{gathered}
\frac{A_{H \downarrow}}{\omega-\epsilon_{\downarrow}+\alpha^{2}\left(t-\epsilon_{+} A_{H \downarrow}\right)+\mathrm{i} \frac{\Gamma_{p \downarrow}}{2}-\Sigma_{\downarrow}^{\operatorname{diag}}(\omega)-\Sigma_{\downarrow}^{\text {mix }}(\omega)}, \\
\Sigma_{\downarrow}^{\operatorname{diag}}(\omega)=\frac{\alpha^{4} \epsilon_{-}^{2} A_{H \downarrow}}{\omega+\epsilon_{\downarrow}-\alpha^{2}\left(t+\epsilon_{+}\right)+\mathrm{i} \frac{\Gamma_{p \downarrow}}{2}}, \\
\Sigma_{\downarrow}^{\text {mix }}(\omega)=\frac{\alpha^{2} \beta^{2} t^{2}}{\omega-\epsilon_{\uparrow}+\beta^{2}\left(t-\epsilon_{+}\right)+\mathrm{i} \frac{\Gamma_{p \uparrow}}{2}}, \\
A_{H \downarrow}=\frac{\omega-\epsilon_{\downarrow}-U\left(1-\left\langle n_{\uparrow}\right\rangle\right)+\alpha^{2} t+\mathrm{i} \frac{\Gamma_{p \downarrow}}{2}}{\omega-\epsilon_{\downarrow}-U+\alpha^{2} t+\mathrm{i} \frac{\Gamma_{p \downarrow}}{2}},
\end{gathered}
$$

where we have introduced the notation: $\alpha \equiv \cos (\theta / 4)$, $\beta \equiv \sin (\theta / 4), t \equiv\left(t_{L}+t_{R}\right) \cos (\Delta \phi / 2), \epsilon_{\mp} \equiv\left(\epsilon_{L}^{2} \mp \epsilon_{R}^{2}\right) / 2 \omega$ and $\epsilon_{\downarrow \mp}=\epsilon_{\downarrow}-\alpha^{2}\left(t \mp \epsilon_{+}\right), \epsilon_{\uparrow \mp}=\epsilon_{\uparrow}-\beta^{2}\left(t \mp \epsilon_{+}\right)$. The corresponding spin-up Green function $G_{\uparrow}$, can be obtained from Eq. (7) by the exchange of spin indices $\downarrow \rightleftarrows \uparrow$ and $\alpha \rightleftarrows \beta$. In the derivation we retained only the first terms $\Sigma_{\downarrow}^{\text {diag }}$ and $\Sigma_{\downarrow}^{\text {mix }}$ of the selfenergy, diagonal in spin indices and mixing the spin indices, respectively. Because the dot is only weakly coupled to the tunnel electrode in order to avoid Kondo resonance formation, the dot's Green's function is calculated within Hubbard I approximation [7], with $U \gg \Delta$, as follows from the experiments [8-12]. For $U \rightarrow \infty$ one obtains $A_{H \sigma}=1-\left\langle n_{\bar{\sigma}}\right\rangle$.

\section{Numerical results and discussion}

In the present paper we concentrate on the transverse zero-bias conductance (ZBC) of the dot through the tunneling electrode calculated within Meir-Wingreen formalism [13], which probes the density of states of the dot $\rho_{\sigma}(\omega)=-(1 / \pi) \Im G_{\sigma}(\omega)$ through relation (per spin $\left.\sigma\right)$ :

$$
\mathcal{G}_{\sigma}=\frac{e^{2}}{h} \frac{\pi \Gamma_{p \sigma}}{2} \int \mathrm{d} \epsilon\left(-\frac{\partial f(\epsilon)}{\partial \epsilon}\right) \rho_{\sigma}(\epsilon) .
$$

For $T=0 \mathrm{ZBC}$ directly reveals the behavior of the density of states at $\omega=\epsilon_{F}$, where MBS resonances emerge. Firstly let us consider the case of long topological wires, $\epsilon_{L}, \epsilon_{R}=0$, when the MBS adjacent to the dot form the bound fermionic state. For $\Theta=0$, which corresponds to the effectively non-interacting dot [6], only $\epsilon_{\downarrow}$ sub-level is active within the gap and $\epsilon_{\uparrow}$ is pushed well above Fermi level. For the renormalized level $\tilde{\epsilon}_{\downarrow}=$ $\epsilon_{\downarrow}-\left(t_{L}+t_{R}\right) \cos (\Delta \phi / 2)$ in resonance with $\epsilon_{F}$ we get $\rho_{\downarrow}(\omega \rightarrow 0)=\left(1 / \pi \Gamma_{p \downarrow}\right)\left(1-\left\langle n_{\uparrow}\right\rangle\right)$. It gives the unitary 

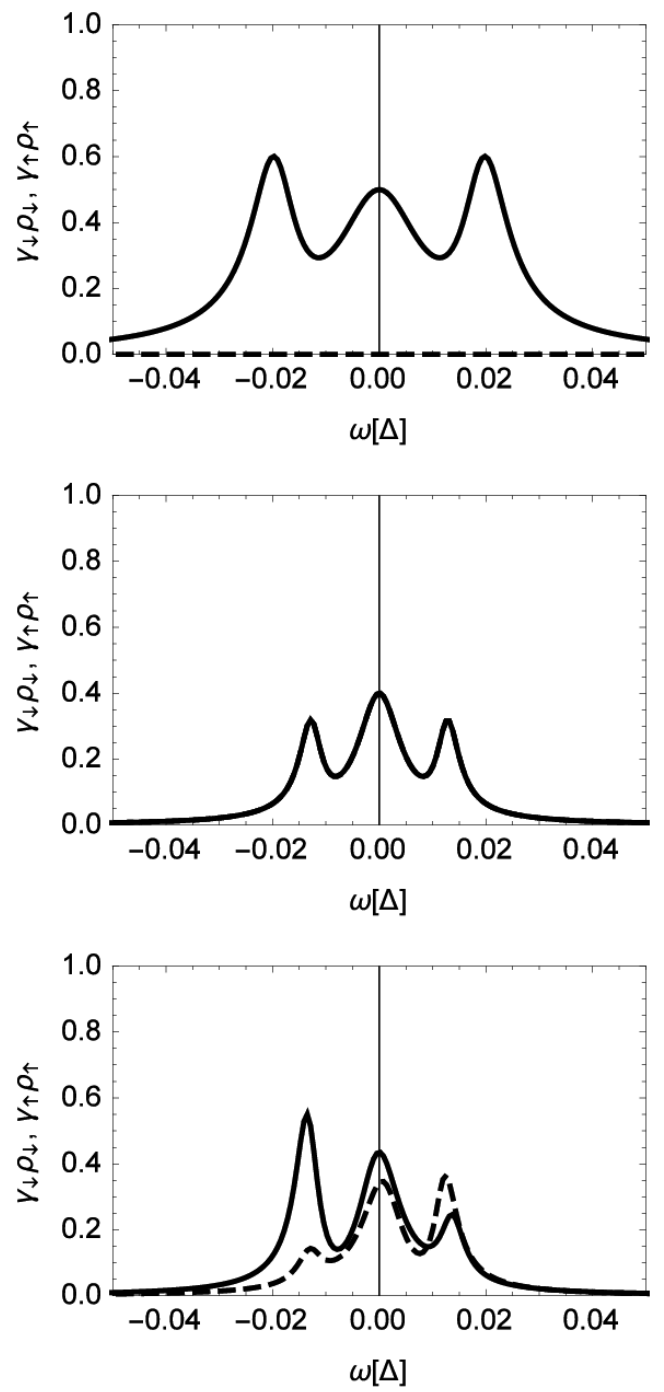

Fig. 2. Spectral densities of the dot (spin $\downarrow$-solid and spin $\uparrow$-dashed curves) multiplied by $\gamma_{\sigma}=\Gamma_{p \sigma} / 2$ for various $\Theta$ angles: 0 -upper panel, $\pi$-middle panel and $0.99 \pi$-lower panel, calculated for $\epsilon_{L}=0.02$ and $\epsilon_{R}=0$, $V_{g}=-2$ for upper panel and $V_{g}=0$ for middle and lower panel. Other input parameters are $\Gamma_{p}=0.02$, $B=2, t_{L}=t_{R}=0.1$ and $\Delta \phi=\pi$.

limit of ZBC in $T=0 ; \mathcal{G}_{\downarrow}=\frac{e^{2}}{h}\left(1-\left\langle n_{\uparrow}\right\rangle\right)$ because of zero occupancy of the upper sub-level $\left\langle n_{\uparrow}\right\rangle=0$.

For one of the hybridizations finite $\epsilon_{\alpha} \neq 0$ and the other $\epsilon_{\alpha^{\prime}}=0$, case depicted in Fig. 2, the unpaired Majorana state produces a characteristic resonance on Fermi level. ZBC in $T=0$ in this case obtained from Eqs. (7) and $(8)$ is $\mathcal{G}_{\sigma}(T=0)=\frac{e^{2}}{h} \frac{1-\left\langle n_{\bar{\sigma}}\right\rangle}{2-\left\langle n_{\bar{\sigma}}\right\rangle}$, which for $\Theta=0$, when $\left\langle n_{\uparrow}\right\rangle=0$ (upper panel of Fig. 2), yields $\mathcal{G}_{\downarrow}(T=0)=\frac{e^{2}}{2 h}$, as for non-interacting $\operatorname{dot}[6]$. For $\Theta=\pi$ we need the values of dot occupancies $\left\langle n_{\downarrow}\right\rangle=\left\langle n_{\uparrow}\right\rangle$, which are calculated selfconsistently by the integration of the spectral density of the dot [14]. Their $V_{g}$ dependencies are practically the same for any arrangement of $\epsilon_{L}$ and $\epsilon_{R}$. In particular for $\epsilon_{L}=\epsilon_{R}=0, \Theta=\pi$ and $\tilde{\epsilon}_{\downarrow / \uparrow}=\epsilon_{F}$ the occupancies can
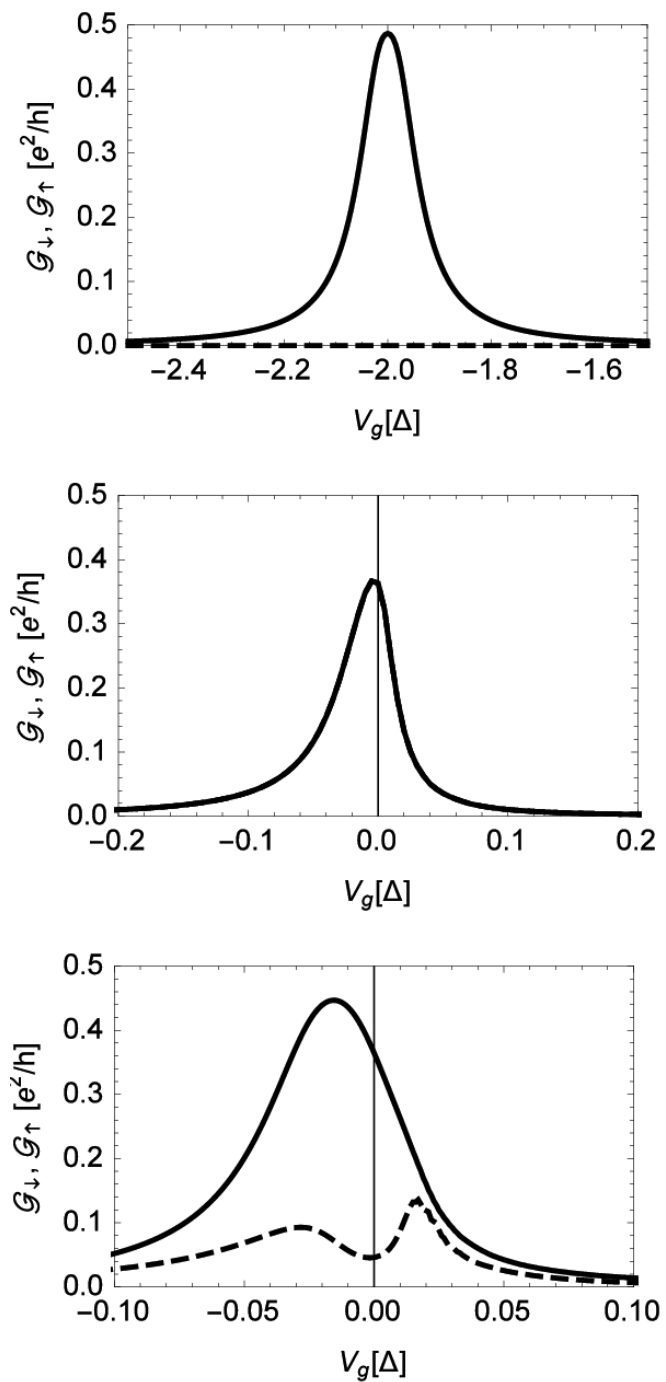

Fig. 3. Zero-bias conductances calculated in $T=0.01$. The arrangement and other calculation parameters are the same as in Fig. 2.

be calculated analytically, yielding $\left\langle n_{\downarrow}\right\rangle=\left\langle n_{\uparrow}\right\rangle=1 / 3$. It implies a unique value of Majorana conductance peak of $\mathcal{G}=(2 / 5) \frac{e^{2}}{h}$ per spin, which is the result of electron interactions inside the dot (see the middle panel of Fig. 2). For finite Zeeman splitting, shown in the lower panel of Fig. 2, the arrangement of $\epsilon_{\downarrow}\left\langle\epsilon_{F}\left\langle\epsilon_{\uparrow}\right.\right.$ implies $\left.\left\langle n_{\downarrow}\right\rangle\right\rangle\left\langle n_{\uparrow}\right\rangle$. It causes the asymmetry of the satellite peaks at $\omega \sim \mp \epsilon_{L}$ and diminishing of the Majorana peak in spin-up sector. Generally the height of the Majorana peak in the spectral density of $\sigma$-sector is scaled approximately by $\sim\left(1-\left\langle n_{\bar{\sigma}}\right\rangle\right)$ and reaches its maximal value of half conductance quantum in spin-down sector for $\Theta=0$, which corresponds to non-interacting dot limit.

In Fig. $3 \mathrm{ZBC}$ dependencies on gate voltage are shown in the same sequence of panels in as in Fig. 2. Electron interactions manifest profoundly in these dependencies. Firstly, the height of the conductance peaks for $\Theta=\pi$ and $0.99 \pi$ are diminished as compared to quasi- 
non-interacting case of $\Theta=0$ as a result of damping of Majorana peak, as discussed above. Secondly, a striking asymmetry of the conductance peaks can be noticed. It is caused by the change with $V_{g}$ of the spectral weight $\sim\left(1-\left\langle n_{\bar{\sigma}}\right\rangle\right)$ of the QD spectral density in the $\sigma$-sector. For negative (positive) gate voltages, where the occupancy is very small (reaches $1 / 2$ ), the spectral weight is larger (smaller). It is worth to emphasize that Majorana peak itself remains symmetric with the change of gate voltage (not shown). The effect of interactions is even more striking for finite Zeeman splitting, lower panel of Fig. 3. The peculiar behavior of occupancies at $V_{g} \sim 0$ in this case, namely large value of $\left\langle n_{\downarrow}\right\rangle$ and small value of $\left\langle n_{\uparrow}\right\rangle$ pushes the spin-up conductance peak, caused by Majorana state, toward positive gate voltages, whereas the peak in spin-down sector is shifted toward negative gate voltages.

\section{References}

[1] S.R. Elliott, Rev. Mod. Physics 87, 137 (2015).

[2] Hao-Hua Sun, Kai-Wen Zhang, Lun-Hui Hu, Chuang Li, Guan-Yong Wang, Hai-Yang Ma, Zhu-An Xu, Chun-Lei Gao, Dan-Dan Guan, Yao-Yi Li, Canhua Liu, Dong Qian, Yi Zhou, Liang Fu, Shao-Chun Li, Fu-Chun Zhang, Jin-Feng Jia, Phys. Rev. Lett. 116 , 257003 (2016).

[3] J.J. He, T.K. Ng, P.A. Lee, K.T. Law, Phys. Rev. Lett. 112, 037001 (2014).
[4] W. Chang, V.E. Manucharyan, T.S. Jespersen, J. Nygard, C.M. Marcus, Phys. Rev. Lett. 110, 217005 (2013).

[5] V. Mourik, K. Zuo, S.M. Vrolov, S.R. Plissard, E.P.A.M. Bakkers, L.P. Kouwenhoven, Science 336 , 1003 (2012).

[6] P. Stefański, J. Phys. Condens. Matter 28, 505301 (2016).

[7] A.C. Hewson, Phys. Rev. 144, 420 (1966).

[8] Y. Kanai, R. S. Deacon, A. Oiwa, K. Yoshida, K. Shibata, K. Hirakawa, S. Tarucha, Phys. Rev. B 82, 054512 (2010).

[9] E.J.H. Lee, X. Jiang, M. Houzet, R. Aguado, C.M. Lieber, S. De Franceschi, Nat. Nanotech. 9, 79 (2014).

[10] M.T. Deng, S. Vaitiekenas, E.B. Hansen, J. Danon, M. Leijnse, K. Flensberg, J. Nygard, P. Krogstrup, C. M. Marcus, 354, 1557 (2016)

[11] M. T. Deng, ScienceC. L. Yu, G. Y. Huang, M. Larsson, P. Caroff, H.Q. Xu, Nano Lett. 12, 6414 (2012).

[12] D. B. Szombati, S. Nadj-Perge, D. Car, S. R. Plissard, E. P. A. M. Bakkers, L. P. Kouwenhoven, Nat. Phys. 12, 568 (2016).

[13] Y. Meir, N.S. Wingreen, Phys. Rev. Lett. 68, 2512 (1992).

[14] P. Stefański, Phys. Rev. B 79, 0859312 (2009). 